\title{
Magnetic Materials and Devices: Research and Applications
}

\author{
Ashutosh Tiwari
}

The history of magnetic materials dates back more than 2,500 years when the magnetic phenomenon was observed in lodestone, a naturally occurring mineral. At that time the only useful application of magnets was in compass needles. Since then, magnetic materials have found applications in a much wider domain. Their use in traditional areas such as electric motors, generators, loudspeakers, magnetic separators, etc. is well known. Lately they have found applications in more advanced fields such as biomedical imaging and drug delivery. However, the one field that has probably been influenced the most is magnetic data storage technology. This technology has been advancing with almost the same pace as the semiconductor industry. A very exciting concept being explored is marrying semiconductor technology and the advanced magnetic data storage and processing technology. This emerging hybrid technology is known as spintronics. Spintronics utilizes the charge as well as the spin degrees of freedom of electrons.

Further, spintronics requires the development of magnetic materials which can efficiently inject spin polarized carriers into the semiconductors. Despite considerable efforts, efficient injection of spins into nonmagnetic semiconductors continues to be a major hurdle in this field. Conventional ferro-magnets such as $\mathrm{Fe}$ and $\mathrm{Ni}$ are not efficient for the above application. This has led to an urgent need for developing new kinds of magnetic materials known as diluted magnetic semiconductors and diluted magnetic insulators. Much research is being carried out worldwide and room temperature ferromagnetism has been reported in many such systems. The three articles assembled in this issue represent the variety of research in this field.

The article by Paul Slusser et al. reports the magnetic behavior of cerium oxide films doped with nonmagnetic dopants. Cerium oxide has a long history in materials science. It is an insulating high-k dielectric material ( $\mathrm{k} \sim 26)$ that is transparent over the visible and infrared bands of the electromagnetic spectrum. The unique redox properties of cerium oxide have led to many different applications including use in solid oxide fuel cell anodes, catalysis, and oxygen sensors. The authors have investigated a relatively unexplored aspect of this material: the magnetic behavior of cerium oxide films when doped with small amounts of copper and zinc. Films with $3 \%$ and $15 \%$ of dopants were deposited by a pulsed laser deposition technique and were thoroughly characterized using $\mathrm{x}$-ray diffraction, x-ray photospectroscopy, and magnetic property measurements. The copper doped films were found to exhibit room temperature ferromagnetism while zinc doped films showed a diamagnetic behavior.

The article by Sudhakar Nori et al. reports room temperature ferromagnetism in vanadium dioxide $\left(\mathrm{VO}_{2}\right)$ films even without any doping. Vanadium dioxide is a very interesting electronic material that undergoes an ultrafast semiconductor-to-metal transition near room temperature. The authors used a pulsed laser deposition system with a $\mathrm{KrF}$ Excimer laser of $248 \mathrm{~nm}(\tau=25$ ns) to prepare $\mathrm{VO}_{2}$ films on sapphire substrates. The room temperature ferromagnetic properties in the system were found to be driven by the stoichiometric defects. Furthermore the authors showed that the ferromagnetism of the epitaxial $\mathrm{VO}_{2}$ films can be switched 'on' and 'off' by altering the cooling ambient conditions.

H.Y. He et al. report the synthesis of M-type $\mathrm{BaAl}_{4} \mathrm{Fe}_{8} \mathrm{O}_{19}$ powders. $\mathrm{M}$ typed hexaferrites are known to possess excellent magnetic properties particularly, a high magnetocrystalline anisotropic field and platelike morphology. This makes these ferrites very useful for applications in microwave materials and microwave absorption. Further enhancement in the magnetocrystalline anisotropy can be achieved by cation substitutions. He et al. used a microemulsion method to make $\mathrm{BaAl}_{4}$ $\mathrm{Fe}_{8} \mathrm{O}_{19}$ powders. The powders were of uniform thin particle morphology, higher shape anisotropy, smaller particle size approaching the theoretical single domain size. It has been shown that the $\mathrm{BaAl}_{4} \mathrm{Fe}_{8} \mathrm{O}_{19}$ powders may be very useful for electromagnetic compatibility and other practical applications at high frequencies.

Ashutosh Tiwari is an associate professor and director of the Nanostructured Materials Research Laboratory in the Department of Materials Science and Engineering, University of Utah, Utah $\mathbf{8 4 1 1 2}$ USA; and the advisor to JOM from the Magnetic Materials Committee of the Electronic, Magnetic \& Photonic Materials Division of TMS.

\section{Ashutosh Tiwari is a TMS Member!}

To read more about him, turn to page 9. To join TMS, visit www.tms.org/Society/Membership.aspx.

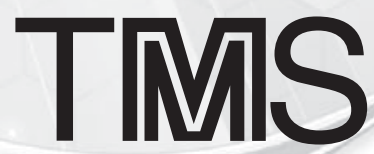

